\title{
Mass Measurement Errors Caused by "Local" Frequency Perturbations in FTICR Mass Spectrometry
}

\author{
Christophe Masselon, Aleksey V. Tolmachev, Gordon A. Anderson, \\ Richard Harkewicz and Richard D. Smith \\ Environmental Molecular Science Laboratory, Pacific Northwest National Laboratory, Richland, Washington, \\ USA
}

One of the key qualities of mass spectrometric measurements for biomolecules is the mass measurement accuracy (MMA) obtained. FTICR presently provides the highest MMA over a broad $m / z$ range. However, due to space charge effects, the achievable MMA crucially depends on the number of ions trapped in the ICR cell for a measurement. Thus, beyond some point, as the effective sensitivity and dynamic range of a measurement increase, MMA tends to decrease. While analyzing deviations from the commonly used calibration law in FTICR we have found systematic errors which are not accounted for by a "global" space charge correction approach. The analysis of these errors and their dependence on charge population and post-excite radius have led us to conclude that each ion cloud experiences a different interaction with other ion clouds. We propose a novel calibration function which is shown to provide an improvement in MMA for all the spectra studied. (J Am Soc Mass Spectrom 2002, 13, 99-106) (c) 2002 American Society for Mass Spectrometry

W ith the increased availability of complete genome sequences, protein identification is now substantially based on searching an appropriate database with physico-chemical data obtained for that protein [1]. Very often, mass spectrometric data from tandem mass spectrometry (MS/MS) experiments using peptides from protein digests are employed [2-4]. One of the aspects of mass spectrometry, which is often viewed as the key to successful protein identification, is mass measurement accuracy (MMA) [5-7]. Increased mass accuracy allows the number of potential masses in a database to be reduced, and sufficiently high MMA may make a peptide unique within the context of a specific proteome [7].

Fourier transform ion cyclotron resonance (FTICR) mass spectrometry currently provides the best achievable mass accuracy over a broad $\mathrm{m} / \mathrm{z}$ range [8-10]. However, the mass accuracy in an FTICR experiment depends crucially on the number of ions used for the measurement $[9,11-18]$. When online separations are used, the analyte ion production rates vary widely, and the ion population in the trap cannot be easily or precisely controlled. Although mass accuracies in the sub-ppm level have been reported using internal calibration [10], external calibration typically doesn't pro-

Published online November 20, 2001

Address reprint requests to Dr. R. D. Smith, Environmental Molecular Science Laboratory, Pacific Northwest National Laboratory, MS K8-98, P.O. Box 999, Richland, WA 99352, USA. E-mail: rd_smith@pnl.gov vide accuracies better than several ppm, unless the ion population (and achievable dynamic range) is severely constrained, or when the ion population for the measurement differs significantly from the ion population used for the calibration [9]. In FTICR, the highest MMA have been obtained with small ion populations, often with the use of summation (or signal averaging) of many spectra and with the application of internal calibration. However, if one desires a large dynamic range, large trapped ion populations are desired, which irrevocably cause relatively large space charge induced frequency shifts, and poorer MMA.

The widely varying ion populations that result from online separation constitute the greatest challenge. The difficulties for large ion populations in FTICR arise because of Coulomb mediated interactions between the different ions present in the cell (and their interactions with their image charge on the detection electrodes), which cause variations in measured frequencies [1418]. It has recently been demonstrated that the frequency shifts induced by coulombic interactions can be compensated for by correcting the detected frequencies, so as to align the deconvoluted spectrum of multiple charge states of the same peptide or protein [19]. This approach provides most of the advantages associated with internal calibration without its disadvantages. This procedure has allowed a significant improvement in mass accuracy for peptides in LC-FTICR experiments, but the mass accuracy realized still plateaus at the few 
ppm level because of the large variations in space charge effects between spectra.

Standard calibration procedures for FTICR have, up to now, incorporated the space charge effect as a global effect resulting only from the number of charges in the trap. However, some frequency perturbations are known to depend on the cyclotron frequency spacing between ions, e.g., the "peak-coalescence" phenomenon [20]. Furthermore, some earlier work has invoked "indirect" coulombic mechanisms to explain why the systematic errors in mass measurement increase with ion number $[21 a, b]$. It is clear that the contribution of such small effects is obscured by the global space charge effect, and while widely recognized, little actual experimental evidence of "local" frequency perturbations has been reported [21a, b, c].

In FTICR, the measured quantity is the effective (cyclotron) frequency of the ions, $f$. This frequency is then converted to an $\mathrm{m} / \mathrm{z}$ value using a calibration function. The most widely used calibration function is:

$$
m / z=\frac{A}{f}+\frac{B}{f^{2}}
$$

This calibration law (Eq 1) was originally derived by Ledford et al. [11] using results from Jeffries et al. [12] and McIver and coworkers [13]. According to this derivation the second term, $B / f^{2}$, accounts for both the DC trapping field and the influence of the space charge. The space charge is assumed to be generated by all ion species present in the ICR cell during collection of the time domain signal. The two calibration coefficients $A$ and $B$ account for factors important for the FTICR mass measurement, i.e., magnetic field strength, and radial components of the trapping DC electrostatic field and the space charge field. Although an additional thirdorder frequency term can be added to the calibration function (Eq 1), there are no quantitative reports on its importance for improved calibration.

We have evaluated the quality of the internal calibration for spectra taken under various experimental conditions in order to determine their influence on mass measurement accuracy. In this process, we have observed systematic errors which could not be compensated for by using a "global space charge" calibration approach. We discuss the effects of ion population and excitation radius regarding these errors, and the possible origin of those "local" frequency perturbations. Given the systematic nature of these errors, we have attempted to empirically compensate for them by using an additional calibration term based upon a simple physical concept.

\section{Experimental}

Perfluoro-alkylphosphazine (Ultramark 1621) was purchased from Thermoquest (San Jose, CA) and used without further purification. The polymer was diluted in acetonitrile to a concentration of $0.002 \% \mathrm{vol} / \mathrm{vol}$. The solution was spiked with $\mathrm{NaOH}\left(20 \mathrm{mM}\right.$ in $\left.\mathrm{H}_{2} \mathrm{O}\right)$ to produce $\mathrm{Na}$ adducts. $\mathrm{K}$ adducts arose naturally from contamination of the solution (e.g., by ambient dust). The polymer solution was introduced to the electrospray ionization (ESI) source at a rate of $0.3 \mu \mathrm{l} / \mathrm{min}$ using a Harvard Apparatus (Holliston, MA) Model 22 syringe-pump.

All experiments were performed using an 11.5 tesla FTICR mass spectrometer controlled by an Odyssey (Finnigan, Madison, WI) data station, and equipped with an external electrospray ion source and an elongated cylindrical open-ended cell, described in detail elsewhere [22].

$\mathrm{A}+2 \mathrm{kV}$ voltage was applied to the ESI emitter, and charged species were injected through a $500 \mu \mathrm{m}$ diameter heated metal capillary maintained at $160^{\circ} \mathrm{C}$. At the exit of the metal capillary, the ion beam was focused to the entrance of a quadrupole ion guide. The ions were accumulated for a period of 200 to $600 \mathrm{~ms}$ (see text) in an external storage quadrupole before transfer to the FTICR cell. After transfer, ions were cooled by a pulse of $\mathrm{N}_{2}$ gas and excited by either a chirp or a stored waveform inverse fourier transform (SWIFT) excitation [23]. The ion signal was digitized at a $761,904 \mathrm{~Hz}$ acquisition frequency for $688 \mathrm{~ms}$ (512 $\mathrm{Kb}$ data points). The resulting transient was zero-filled twice before Fourier transformation and the peaks were picked in the frequency domain using a 3 points quadratic approximation which was previously shown to provide the best MMA [10]. Only monoisotopic peaks with signal to noise $>3$ and relative intensity $>5 \%$ were used to generate the calibration. Data analysis was performed using the ICR-2LS software package developed in our laboratory [24].

\section{Results and Discussion}

\section{Evidence for Local Frequency Perturbations in FT-ICR}

The first experiments were performed to study the effect of ion population on the internal calibration. Series of single spectra (no signal averaging) were recorded for different ion populations of perfluoroalkylphosphazine (Ultramark). A typical mass spectrum of Ultramark (Figure 1a) shows the molecular weight distribution of the polymer and the sodium and potassium adduction. Monoisotopic masses for the protonated, sodiated and potassiated ions of the polymer were used for calibration.

The ion population could not be easily determined experimentally (based on TIC measured from the ICR spectra), since it did not vary linearly with the accumulation time. However, the ion population did vary monotonically with the external ion accumulation time in our experiments. The total ion population in the ICR cell was thus varied by using 200, 400, and $600 \mathrm{~ms}$ external accumulation times. 


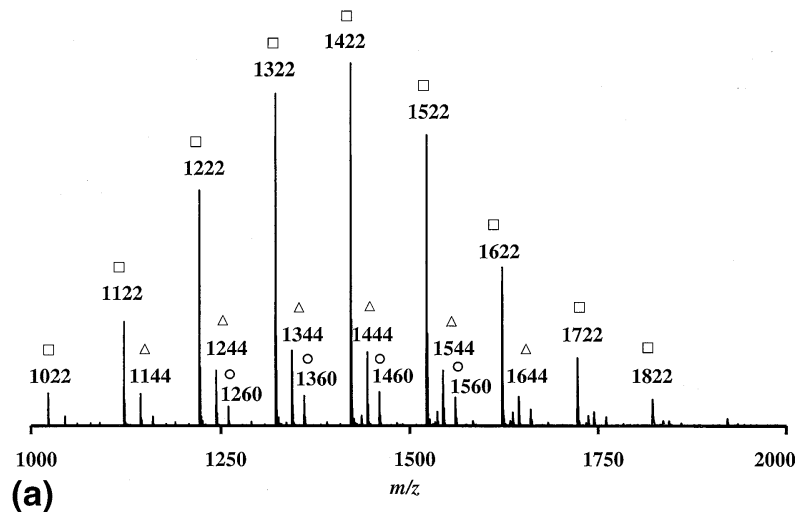

(a)

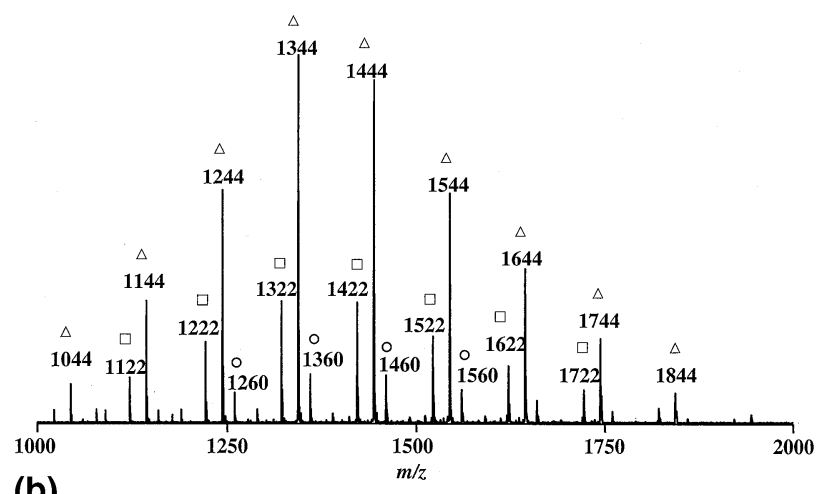

(b)

Figure 1. FTCIR mass spectrum for Ultramark showing the protonated (open square), sodiated (open triangle), and potassiated (open circle) polymer ion distributions; for (a) a sample with a low sodium concentration, and (b) a sample with a higher sodium concentration.

The aim of our study was to analyze deviations from the calibration function (Eq 1) under conditions typical for LC-FTICR measurements, i.e., where the highest possible mass accuracy is desirable in a wide mass range and for widely varying ion populations. Electrospray spectra of Ultramark solutions were used to obtain accurate mass-reference values in the mass range $1000-1800 \mathrm{u}$. To test the calibration function (Eq 1) we have used the linearized form:

$$
F(x)=A+B \cdot x ; F(x) \equiv m / z \cdot f, x \equiv \frac{1}{f}
$$

Each peak of known $m / z$ yields a point on the $F(x)$ plot. Using the Ultramark $[\mathrm{M}+\mathrm{H}]^{+}$peaks together with its $\mathrm{Na}^{+}$and $\mathrm{K}^{+}$adducts, we obtained $\sim 20$ points on such a plot (see Figure 2). Deviations from the calibration function (Eq 1) were then analyzed by calculating a linear regression, which results in accurate $A$ and $B$ calibration coefficients and also yields the correlation coefficient, showing the quality of the linear approxi-

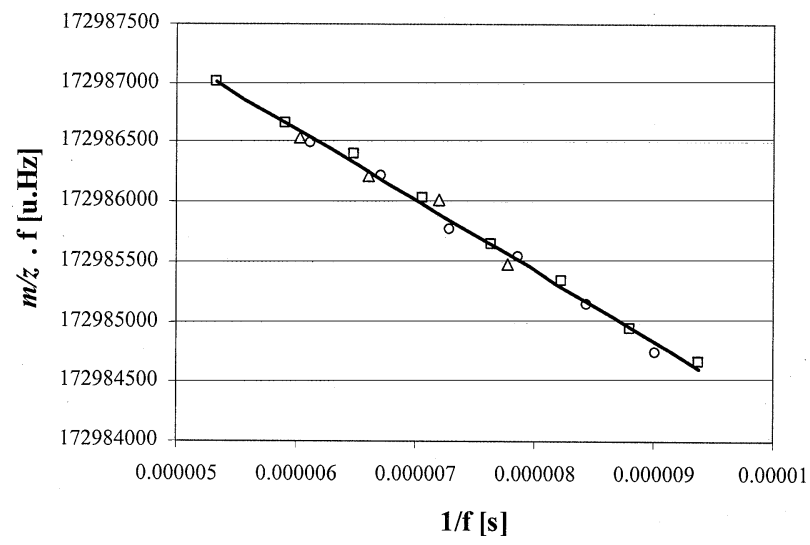

Figure 2. $(\mathrm{m} / \mathrm{z}) f$ versus inverse of frequency plot for an FTICR spectrum of an Ultramark calibration solution showing the linear fit using calibration Eq 2. The points represent known $\mathrm{m} / \mathrm{z}$ values of (open square) $[\mathrm{M}+\mathrm{H}]^{+}$ions, (open traingle) $[\mathrm{M}+\mathrm{Na}]^{+}$ions, and (open circle) $[\mathrm{M}+\mathrm{K}]^{+}$ions of the polymer. mation. Ideally, the correlation coefficient should be close to 1 , and errors should be confined to the ppm level.

By plotting the $(m / z \cdot f)$ versus $(1 / f)$ graph for the series of data files obtained from these measurements, we observed that some masses were systematically over- or under-corrected. Figure 3 shows the mass errors obtained for an internal calibration of two spectra acquired at 400 and $600 \mathrm{~ms}$ accumulation respectively; the error bars indicate the $95 \%$ confidence interval for 10 measurements obtained under the same conditions. There is no obvious trend of a mass dependent deviation from linearity. Figure 4 shows the $(\mathrm{m} / \mathrm{z} \cdot \mathrm{f})$ versus $(1 / f)$ plot for three spectra taken at different accumulation times. It is evident, that a "global" space charge effect is present, thus increasing the absolute value of the slope of the plot ( $B$ coefficient). At the same time, the errors become more pronounced when the ion population in the ICR trap increases, and errors of more than 3 ppm (even using internal calibration!) were obtained for the $600 \mathrm{~ms}$ accumulation experiment, with

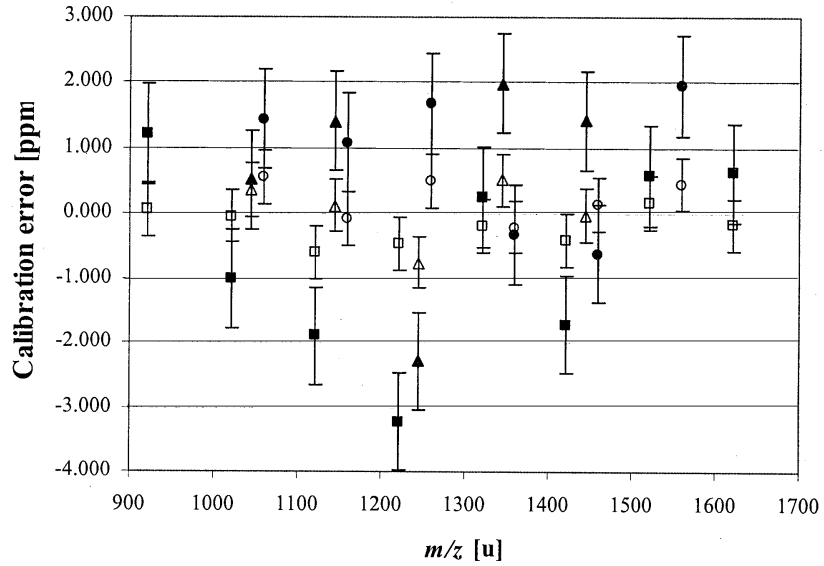

Figure 3. Calibration errors obtained from the linear fit from Eq (2) versus $\mathrm{m} / \mathrm{z}$ for external accumulation times $400 \mathrm{~ms}$ (open symbols) and $600 \mathrm{~ms}$ (filled symbols) using chirp excitation (symbols as in Figure 2). Error bars represent 95\% confidence intervals based on 10 measurements. 


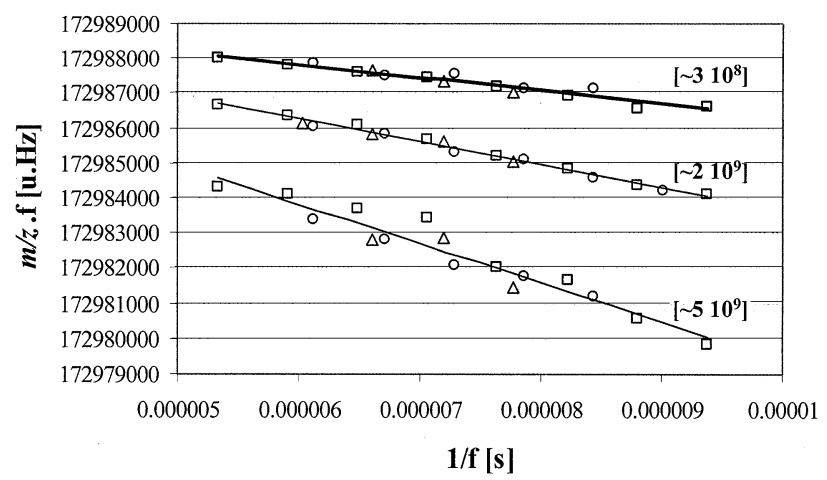

Figure 4. Calibrations obtained for three ion populations resulting from different external accumulation times, TIC (arbitrary units) indicated between brackets (symbols as in Figure 2). Correlation coefficients are $0.94,0.99$, and 0.91 for the 200, 400, and 600 $\mathrm{ms}$ accumulation times, respectively.

correlation coefficients for the calibration function decreasing to 0.91 (see Figure 4). The striking fact is that while the frequencies were measured to a precision better than $0.25 \mathrm{~Hz}$ (corresponding to mass errors of $\sim 1$ ppm, see error bars on Figure 3), the calibration correction was ineffective.

It appears from these measurements that either the ion population has to be carefully controlled in order to avoid large systematic errors even with internal calibration, or that improved calibration methods are required. At higher population, space charge effects cause larger mass errors, and at too low ion population, the contribution of the noise ultimately becomes limiting and degrades the mass accuracy as well as the dynamic range.

A possible origin for the systematic errors determined previously could be due to variations in excite radii for different $\mathrm{m} / \mathrm{z}$, since the chirp excite waveforms used for excitation are known not to provide an optimally flat excitation spectrum [23]. In order to test this hypothesis, additional experiments were performed using SWIFT excitation, which provides a flater excitation over the $\mathrm{m} / \mathrm{z}$ range of interest [23].

A SWIFT excitation waveform has been calculated to excite all ions in the $\mathrm{m} / \mathrm{z}$ range of interest. An external accumulation of $400 \mathrm{~ms}$ was used for these experiments since it provided the lowest overall error in the previous measurements. The plot of the mass errors versus $\mathrm{m} / \mathrm{z}$ is shown in Figure 5a for a series of 10 spectra taken for the same conditions (10 single acquisitions at a post-excite radius of $\sim 1.26 \mathrm{~cm}$ ). As can be seen from this plot, the use of SWIFT excitation similarly reveals systematic errors in mass measurements (which were smaller in amplitude). It is worth noting that the error bars were also smaller because of the better controlled post-excite radius.

The most striking observation from Figure $5 a$ is the clear pattern in the plot of the errors versus $\mathrm{m} / \mathrm{z}$. The peaks in the center of the spectrum, where the abundances were greater, had larger errors than the peaks at the extremes, and their errors were consistent with the intensities when taken "locally", i.e., lower abundance ions were found to have errors skewed on one side of the calibration and high abundance ions on the other side. This implies that the uncorrected cyclotron frequencies for higher abundance ions were too high and frequencies for low abundance ions were too low. However, the errors were not found to be highly correlated with the abundances of the individual ions; for instance, the mass of an $[\mathrm{M}+\mathrm{H}]^{+}$ion at the edge of the distribution was better determined than an $[\mathrm{M}+$ $\mathrm{Na}]^{+}$in the center of the spectrum although its intensity was smaller than the latter.

These experiments were repeated using different SWIFT excitation radii, and we have observed that the systematic errors become larger for smaller excitation radii (see Figure 6). The correlation coefficient dropped
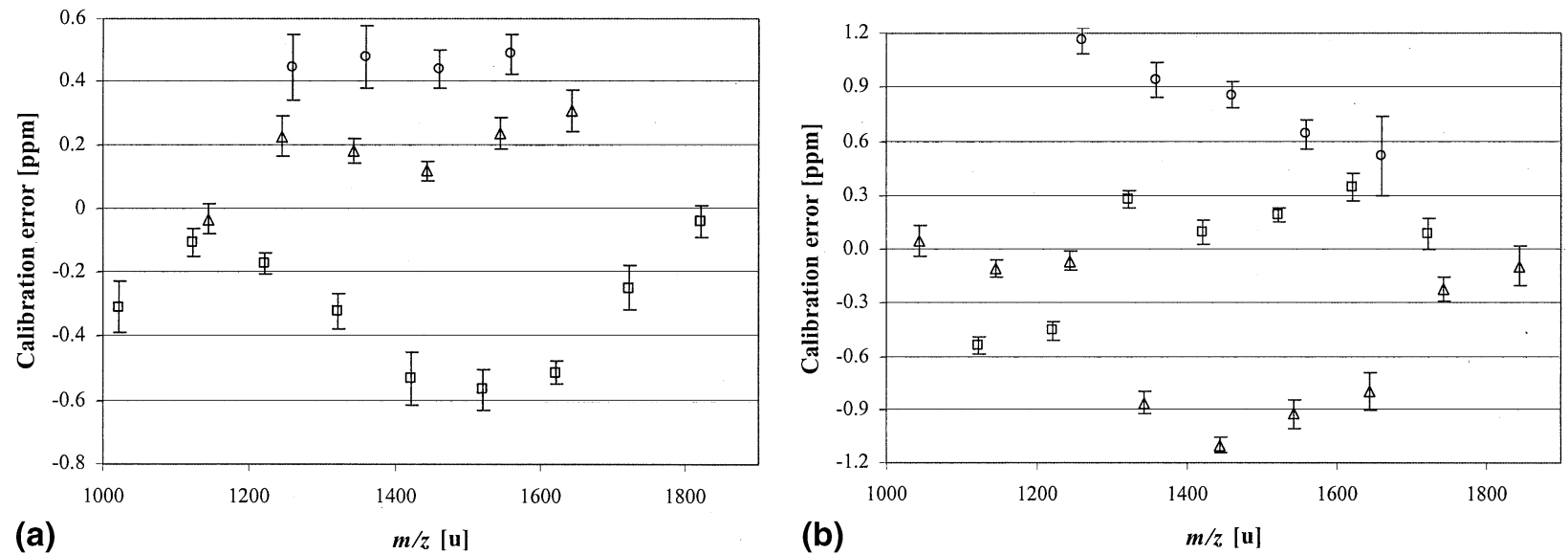

Figure 5. Calibration errors obtained from a linear fit using Eq (2) versus $\mathrm{m} / \mathrm{z}$ for a SWIFT excitation to a radius $1.26 \mathrm{~cm}$ (symbols as in Figure 2). Note the repartition of the points around the calibration with the masses of protonated species always underestimated, and those of sodiated and potassiated species always overestimated (a). A reversal of this trend is observed when a higher sodium concentration is used (b). Error bars represent 95\% confidence intervals based on 10 measurements. 


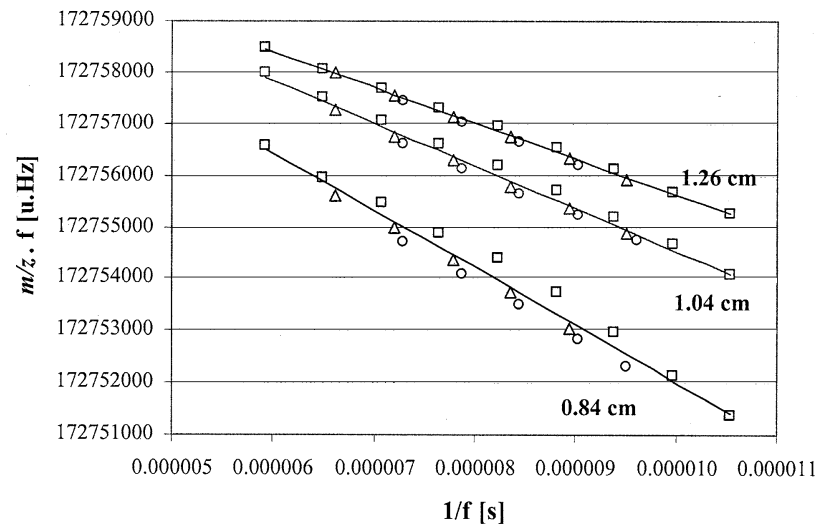

Figure 6. Calibrations obtained for three post-excite radii after SWIFT excitation, post-excite radii indicated between brackets (symbols as in Figure 2).

from 0.98 to 0.80 for a decrease in radius from $1.26 \mathrm{~cm}$ to $0.84 \mathrm{~cm}$.

These observations, together with the fact that those errors increased with ion abundances, suggest that the observed effects originate from Coulomb mediated interactions. The reproducible nature of the observed errors also suggests that individual ion clouds in the ICR cell experience qualitatively different interactions with the other ion clouds.

To further investigate this issue, we have studied a sample with higher $\mathrm{NaOH}$ concentration resulting in a distorted distribution of ionic species (see Figure 1b) compared to our initial experiments. Analysis of the calibration errors revealed similar behavior. The most abundant ions (in this case the $[\mathrm{M}+\mathrm{Na}$ ] species) were primarily shifted to higher frequency relative to the calibration, whereas the lower abundance species ([M + $\mathrm{H}]$ and $[\mathrm{M}+\mathrm{K}]$ ) were shifted to lower frequency (see Figure $5 b$ ). These data show that the observed frequency shifts are related to the number of ions for each species (signal intensities) and not to the nature of a specific ionic species.

\section{Correction for the Frequency Shifts}

The systematic behavior observed for the frequency shifts may be used to correct these shifts and to improve the mass measurement accuracy. As discussed above, the SWIFT-excite data show a correlation between peak intensities and the frequency shift magnitudes. More intense peaks have positive frequency shifts, and less intense ones reveal negative frequency shifts. In terms of measured $\mathrm{m} / \mathrm{z}$ (Figure $5 \mathrm{a}$ ) all intense peaks have negative mass errors (protonated ions, squares), and lower abundance peaks have positive mass deviations from the calibration line $\left(\mathrm{Na}^{+}\right.$and $\mathrm{K}^{+}$ions, triangles and circles).

These deviations from the standard calibration law may be interpreted in terms of space charge induced frequency shifts. The role of the ion space charge becomes increasingly important for LC-FTICR studies because both the total ion population and specific ion abundances may vary in a wide range. A systematic study of the mass accuracy under such conditions [9] has demonstrated that the frequency correction based on the overall intensity can improve the FTICR mass measurement accuracy over a wide mass range for externally calibrated spectra.

Our detailed measurements of the frequency shifts demonstrate that an approach considering only the total ion intensity is insufficient, and individual peak intensities must be taken into account if higher MMA is to be realized. Positive frequency shifts for more intense peaks may indicate that ion clouds having greater numbers of charges experience reduced space charge effects. This is consistent with a concept first described by Wineland and Dehmelt [14, 25] that the space charge associated with an ion cloud consisting of particles of a single $\mathrm{m} / \mathrm{z}$ cannot influence the center-of-mass motion of this cloud. Thus, if both ion excitation and detection of the ion cloud can be described in terms of the center-of-mass motion, the frequency shift due to the ion cloud itself must vanish, and only other $\mathrm{m} / \mathrm{z}$ clouds will contribute to the space-charge induced frequency shift.

Another possible and related mechanism may be associated to the phase-locking phenomenon [26, 27]. Although considered mostly for two different ion species of close $m / z$, the same phenomenon is responsible for the long term coherence (ion cloud stability) of ions of the same $m / z$. That provides the basis for the very high mass resolution capability of FTICR. Under these conditions one can expect that the ion cloud motion will resemble the motion of a single macro-ion and will experience Coulomb interaction mostly from other $\mathrm{m} / \mathrm{z}$ ions. To analyze the problem in detail, additional experimental and theoretical investigations are needed and are being pursued. Here we propose empirical relationships providing a description of the observed behavior.

According to the original derivation, the coefficient $B$ in the calibration function $(\mathrm{Eq} 1)$ can be divided into two parts, one for the magnetron motion, $B_{\text {trap }}$, and one for the total space charge correction, $B_{\text {total }}^{S C}$ :

$$
\begin{aligned}
B & =B_{\text {trap }}+B_{\text {total }}^{S C} B_{\text {total }}^{S C} \\
& =K \cdot I_{\text {total }} ; I_{\text {total }} \\
& =\Sigma I_{i}
\end{aligned}
$$

It is assumed here that the space-charge term is proportional to the total ion population, and can be expressed in terms of the total ion intensity of a mass spectrum $I_{\text {total }}$ multiplied by some constant $K$. Here we use a feature of FTICR magnitude-mode mass spectra: to a good approximation peak intensities $\left(I_{i}\right)$ are proportional to the total ion charges of corresponding $(\mathrm{m} / \mathrm{z})_{\mathrm{i}}$ ions [28]. The space charge term $B^{S C}$ total is negative, because the space charge influence results in decreasing 
Table 1. Mass measurement accuracy obtained in various conditions from the summation of 10 time-domain transient: Comparison of the common calibration law, Eq 1, and the modified calibration law, Eq 6

\begin{tabular}{lrcccc}
\hline $\begin{array}{l}\text { Excite } \\
\text { radius }\end{array}$ & $\begin{array}{c}\text { Mass } \\
\text { range, Da }\end{array}$ & $\begin{array}{c}\text { Number of } \\
\text { peaks }^{\mathrm{a}}, N\end{array}$ & $\begin{array}{c}\text { Mass error } \\
\varepsilon_{0}, \mathrm{mDa}\end{array}$ & $\begin{array}{c}\text { Corrected error }^{\mathrm{b}} \\
\varepsilon^{\mathrm{mDa}}\end{array}$ \\
\hline \hline $1.3 \mathrm{~cm}$ & $0-2000$ & 19 & 0.514 & 0.329 \\
& $1200-1700$ & 14 & 0.567 & 0.200 \\
$\varepsilon_{0} / \varepsilon$
\end{tabular}

${ }^{a} \mathrm{~A}$ dynamic range of 0.05 has been used for choosing the reference peaks. i.e., only peaks of amplitude greater than 0.05 of the most abundant peak were used.

${ }^{b}$ Obtained using Eq (1) (see text).

'Obtained using Eq (5) (see text).

* $\mathrm{Na}$ - enriched sample, see Figure $2 \mathrm{~b}$.

the cyclotron frequency. Thus the constant $K$ defined in (Eq 3) is also negative.

For the situation considered above, when clouds of the same $\mathrm{m} / \mathrm{z}$ ions experience only "external" spacecharge influence, we must subtract each peak intensity $I_{i}$ from $I_{\text {total }}$ :

$$
B_{i}=K \cdot\left(I_{\text {total }}-I_{i}\right)=B_{\text {total }}^{S C}-K \cdot I_{i}
$$

Here $i$ is an index designating each separate $m / z$ ion species as $(\mathrm{m} / \mathrm{z})_{i}$. The corrected space charge term $B_{i}$ in Eq 4 takes into account the influence of all ion species except the current $i$-th specie. We can now regroup terms to separate those having the index $i$, arriving at the following relationship:

$$
(m / z)_{i}=\frac{A}{f}+\frac{B_{\text {trap }}+B_{\text {total }}^{S C}}{f^{2}}-\frac{K \cdot I_{i}}{f^{2}}
$$

This relationship can be used as a corrected calibration equation of the following form:

$$
(m / z)_{i}=\frac{A}{f}+\frac{B}{f^{2}}+\frac{C \cdot I_{i}}{f^{2}}
$$

The equation differs from the common calibration law (Eq 1) by the additional term $C \cdot I_{i} / f^{2}$ that takes into account the individual peak intensities $I_{i}$. The coefficient $B$ can be interpreted as the sum of the magnetron and space charge terms, as it is for the common calibration. Both factors decrease the effective cyclotron frequency, so the $B$ coefficient is always negative. We introduced here a third calibration coefficient $(C=-K)$, which is expected to have a positive sign. Thus for each ion species " $\mathrm{i}$ ", the calibration takes into account not the total space charge but the space charge induced by all other ions but the ions of type " $\mathrm{i}$ ".
The calibration coefficients $A, B$, and $C$ are independent of the index $i$. Thus to calibrate a mass spectrum using $\mathrm{Eq}(6)$, one needs at least three reference peaks, i.e., peaks with accurately defined frequencies (attributed to known $\mathrm{m} / \mathrm{z}$ values). When more than three reference peaks are present, as in the case of our Ultramark spectra considered above, it is possible to use the least-squares fitting procedure (LSQ) to obtain the coefficients that give the best overall fit to the peaks used for calibration. The mass of an unknown peak can then be computed based on its measured frequency and intensity using Eq (6) and the parameters $\mathrm{A}, \mathrm{B}$, and C estimated from the calibrants.

Such LSQ calibration, based on the modified calibration function (Eq 6), has been applied to the Ultramark spectra discussed above. To evaluate the overall accuracy of the mass calibration we used an average squared mass error $\varepsilon$, calculated as follows:

$$
\varepsilon=\sqrt{\frac{1}{N} \sum \delta m_{i}{ }^{2}} ; \quad \delta m_{i}=F\left(f_{i}, I_{i}\right)-(m / z)_{i}
$$

Here $N$ is the number of reference peaks used for the calibration; each mass error $\delta m_{i}$ is calculated as the difference between the calibration function $F\left(f_{i}, I_{i}\right)$, estimated using Eq (6) for the corresponding peak frequency $f_{i}$ and intensity $I_{i}$, and the accurate value $(m / z)_{i}$.

Table 1 lists the calibration mass errors obtained for mass spectra with estimated excitation radii of 1.3, 1.0, and $0.84 \mathrm{~cm}$ (see discussion above concerning the SWIFT-excite spectra). Three different mass ranges were considered for each spectrum. For comparison we also calculated the corresponding mass error without correction $\left(\varepsilon_{0}\right)$ obtained using the same procedure, with the common calibration function $(\mathrm{Eq} 1)$ instead of function (Eq 6). All errors are expressed in $\mathrm{mDa}$, which 


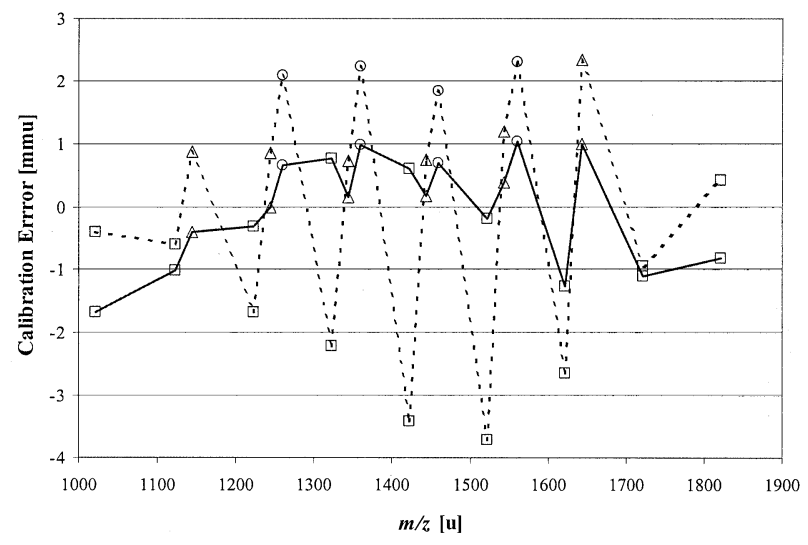

Figure 7. Calibration errors for the standard calibration (dashed line) function and for the calibration using Eq (6) (solid line). Summation of 10 spectra using SWIFT excitation to a $0.84 \mathrm{~cm}$ post-excite cyclotron radius (symbols as in Figure 2). Lines are only represented to guide the eye.

roughly correspond to relative errors in ppm for mass values around $1000 \mathrm{Da}$. Each spectrum was obtained by accumulation of 10 single time domains. (Similar results have been obtained for single spectra, with only slightly larger absolute values of the mass errors due to the lower $\mathrm{S} / \mathrm{N}$ ). As discussed above, deviations from the common calibration function (Eq 1) are larger for spectra having smaller excitation radii. The modified calibration function (Eq 6) results in noticeably reduced mass errors. The observed improvement was greater when applied over a limited mass interval (e.g., 1300$1500 \mathrm{Da}$ ), in the central region of the mass spectra. The relative improvement resulting from the modified calibration function, shown in the column " $\varepsilon_{0} / \varepsilon^{\prime}$, is typically larger for spectra having lower excitation radius. This is consistent with the above considerations, because the space charge induced frequency shifts are expected to decrease with an increase in the ion cloud post-excite radius.

To test the assumption that the frequency shifts are related to peak intensities and not to the type of ion species, we have prepared a sample in which $[\mathrm{M}+$ $\mathrm{Na}]^{+}$peaks dominated over the $[\mathrm{M}+\mathrm{H}]^{+}$and $[\mathrm{M}+$ $\mathrm{K}^{+}$peaks (Figure $1 \mathrm{~b}$ ). The modified calibration function (Eq 6) applied to these spectra resulted in the similar mass accuracy improvement (see Table 1).

The calibration coefficients obtained for the modified calibration function (Eq 6) correspond qualitatively to the simple model advanced in the derivation of the modified calibration function. The $A$ coefficient is positive and corresponds to the magnetic field of $11.5 \mathrm{~T}$. Essentially the same value of $A$ was obtained using the common calibration function (Eq 1 ). The $B$ coefficient is always negative and the $C$ coefficient is positive, as expected.

Mass errors for each reference peak obtained for the common and modified calibration functions are plotted in Figure 7. It is obvious that the modified calibration function (Eq 6), when applied over the whole mass range, does not fully compensate the systematic frequency shifts. This indicates that the modified calibration function (Eq 6), and the simple physical concept behind it, can be further improved. The deviation from linearity after correction may in fact be related to a post-excite radius dependence on $\mathrm{m} / \mathrm{z}$. Studies in this direction are in progress and we plan to evaluate the modified calibration function for FTICR spectra obtained in biological applications, and where improvements in MMA could serve to greatly advance many important uses of mass spectrometry.

\section{Conclusions}

While analyzing deviations from the commonly used calibration law in FTICR, we have found systematic errors which are not corrected by this approach. The conventional calibration equation corrects for "global" space charge effects where it is assumed that all species are shifted in the same fashion. The reproducibility of the observed errors and their dependence on individual ion populations and post-excite radius have led us to conclude that each individual ion cloud experiences somewhat different interactions with other ion clouds. This affects its particular cyclotron frequency and results in mass measurement error. We have found that the errors' absolute values correlate with individual peak intensities.

A relatively simple corrected calibration function has been proposed, based on the assumption that the space charge induced frequency shift of each $\mathrm{m} / \mathrm{z}$ ion cloud is defined by the total ion population minus it's own ion charge. The corrected calibration function results in improved mass accuracy for all experimental mass spectra studied. The correction is especially efficient when applied over the central region of a mass spectrum. While somewhat more demanding computationaly, such improvement promises to significantly impact many areas of application where MMA is of great importance, such as proteomics [7]. Further studies will focus on more detailed experimental and theoretical investigation of deviations from the common mass calibration function.

\section{Acknowledgments}

The authors gratefully acknowledge Drs. V. S. Rakov, L. PasaTolic, E. N. Nikolaev, M. V. Gorshkov, and M. Belov for helpful discussions and comments. We thank the National Cancer Institute (Grant CA81654) and U.S. Department of Energy, Office of Biological and Environmental Research, for support of portions of this research. Pacific Northwest National Laboratory is operated by Battelle Memorial Institute for the U.S. Department of Energy through Contract DE-AC06- 76RLO 1830.

\section{References}

1. Yates, J. R., III; McCormack, A. L.; Eng, J. Mining Genomes with MS. Anal. Chem. 1996, 68, 534A-540A. 
2. Mann, M.; Hojrup, P.; Roepstroff, P. Use of Mass Spectrometric Molecular Weight Information to Identify Proteins in Sequence Databases. Biol. Mass Spectrom. 1993, 22, 338-345.

3. James, P.; Quadroni, M.; Carafoli, E.; Gonnet, G. Protein Identification by Mass Profile Fingerprinting. Biochem. Biophys. Res. Commun. 1993, 195, 58-64.

4. Yates, J. R., III; Speicher, S.; Griffin, P. R.; Hunkapiller, T. Peptide Mass Maps: A Highly Informative Approach to Protein Identification. Anal. Biochem. 1993, 214, 397-408.

5. Jensen, O. N.; Podtelejnikov, A. V.; Mann, M. Identification of the Components of Simple Protein Mixtures by HighAccuracy Peptide Mass Mapping and Database Searching. Anal. Chem. 1997, 69, 4741-4750.

6. Clauser, K. R.; Baker, P.; Burlingame, A. L. Role of Accurate Mass Measurement ( $\pm 10 \mathrm{ppm})$ in Protein Identification Strategies Employing MS or MS/MS and Database Searching. Anal. Chem. 1999, 71, 2871-2882.

7. Conrads, T. P.; Anderson, G. A.; Veenstra, T. D.; Pasa-Tolic, L.; Smith, R. D. Utility of Accurate Mass Tags for Proteome-Wide Protein Identification. Anal. Chem. 2000, 72, 3349-3354.

8. Gorshkov, M. V.; Guan, S.; Marshall, A. G. Masses of Stable Neon Isotopes Determined at Parts Per Billion Precision by Fourier Transform Ion Cyclotron Resonance Mass Spectrometry. Int. J. Mass Spectrom. Ion Processes 1993, 128, 47-60.

9. Easterling, M. L.; Mize, T. H.; Amster, I. J. Routine Part-PerMillion Mass Accuracy for High-Mass Ions: Space-Charge Effects in MALDI FT-ICR. Anal. Chem. 1999, 71, 624-632.

10. Burton, R. D.; Matuszak, K. P.; Watson, C. H.; Eyler, J. R. Exact Mass Measurement Using a 7 Tesla Fourier Transform Ion Cyclotron Resonance Mass Spectrometer in a Good Laboratory Practice-Regulated Environment. J. Am. Soc. Mass Spectrom. 1999, 10, 1291-1297.

11. Ledford, E. B.; Rempel, D. L.; Gross, M. L. Space Charge Effects in Fourier Transform Mass Spectrometry. Mass Calibration. Anal. Chem. 1984, 56, 2744-2748.

12. Jeffries, J. B.; Barlow, S. E.; Dunn, G. H. Theory of SpaceCharge Shift of Ion Cyclotron Resonance Frequencies. Int. J. Mass Spectrom. Ion Processes 1983, 54, 169-187.

13. Francl, T. J.; Sherman, M. G.; Hunter, R. L.; Locke, M. J.; Bowers, W. D.; McIver, R. T. Experimental Determination of the Effects of Space Charge on Ion Cyclotron Resonance Frequencies. Int. J. Mass Spectrom. Ion Processes 1983, 54, 189-199.

14. Wineland, D. J.; Dehmelt, H. G. Principles of the Stored Ion Calorimeter. J. Appl. Phys. 1975, 46, 919-930.

15. Chen, S.; Comisarow, M. Simple Physical Models for Coulomb-Induced Frequency Shifts and Coulomb-Induced Inhomogeneous Broadening for Like and Unlike Ions in Fourier Transform Ion Cyclotron Resonance Mass Spectrometry. Rapid Commun. Mass Spectrom. 1991, 5, 450-455.

16. Chen, S.; Comisarow, M. Modelling Coulomb Effects in Fourier-Transform Ion Cyclotron Resonance Mass Spectrometry by Charged Disks and Charged Cylinders. Rapid Commun. Mass Spectrom. 1992, 6, 1-3.
17. Xiang, X. Z.; Grosshans, P. B.; Marshall, A. G. Image ChargeInduced Ion-cyclotron Orbital Frequency-Shift for Orthorhombic and Cylindrical FT-ICR Ion Traps. Int. J. Mass Spectrom. Ion Processes 1993, 125, 33-43.

18. Gorshkov, M. V.; Marshall, A. G.; Nikolaev, E. N. Analysis and Elimination of Systematic Errors Originating from Coulomb Mutual Interaction and Image Charge in Fourier Transform Ion Cyclotron Resonance Precise Mass Difference Measurements. J. Am. Soc. Mass Spectrom. 1993, 4, 855-868.

19. Bruce, J. E.; Anderson, G. A.; Brands, M. D.; Pasa-Tolic, L.; Smith, R. D. Obtaining More Accurate Fourier Transform Ion Cyclotron Resonance Mass Measurements Without Internal Standards Using Multiply Charged Ions. J. Am. Soc. Mass Spectrom. 2000, 11, 416-421.

20. Pasa-Tolic, L.; Huang, Y.; Guan, S.; Kim, H. S.; Marshall, A. G. Ultrahigh-Resolution MALDI FTICR Mass Spectra of Peptides. J. Mass Spectrom. 1995, 30, 825-833.

21. (a) Yang S. S., Rempel D. L., Gross M. L. Exact Mass Errors in Fourier Transform Mass Spectrometry: Experimental Evidence for Indirect Coulombic Mechanisms. Proceedings of the 36th ASMS Conference on Mass Spectrometry And Allied Topics; San Francisco, CA, June 1988; pp 586-587. (b) Yang S. S., Rempel D. L., Gross M. L. A Model for the Reduction of Mass Measurement Errors Due to Ion-Ion Interactions in FTMS. Proceedings of the 37th ASMS Conference on Mass Spectrometry And Allied Topics; Miami Beach, FL, May 1989; 1224-1225. (c) Huang J. Y., Tiedemann P. W., Land D. P., McIver R. T., Hemminger J. C. Dynamics of Ion Coupling in an FTMS Ion Trap and Resulting Effects on Mass Spectra, Including Isotope Ratios. Int. J. Mass Spectrom. Ion Proc. 1994, 134(1), 11-21.

22. Udseth H. R., Gorshkov M. V., Belov M. L., Pasa-Tolic L., Bruce J. E., Masselon C. D., Harkewicz R., Anderson G. A., Smith R. D. Proceedings of the 47th ASMS Conference on Mass Spectrometry And Allied Topics; Dallas, TX, June, 1999; pp 1111-1112.

23. Marshall, A.G.; Hendrickson, C.L.; Jackson, G.S. Fourier Transform Ion Cyclotron Resonance Mass Spectrometry: A Primer. Mass Spectrom. Rev. 1998, 17, 1-35.

24. Anderson G.A., Bruce J.E., Eds. ICR-2LS1995. Pacific Northwest National Laboratory: Richland, WA, 1995.

25. Wineland, D. J.; Dehmelt, H. G. Line Shifts and Widths of Axial, Cyclotron and G-2 Resonances in Tailored, Stored Electron (Ion) Cloud. Int. J. Mass Spectrom. Ion Phys. 1975, 16, 338-342.

26. Mitchell, D. W.; Smith, R. D. Cyclotron Motion of Two Coulombically Interacting Ion Clouds with Implications to Fourier-Transform Ion Cyclotron Resonance Mass Spectrometry. Phys. Rev. E. 1995, 52, 4366-4386.

27. Mitchell, D. W.; Smith, R. D. Prediction of a Space Charge Induced Upper Molecular Mass Limit Towards Achieving Unit Mass Resolution in Fourier Transform Ion Cyclotron Resonance Mass Spectrometry. J. Mass Spectrom. 1996, 31, 771-790.

28. Comisarow, M. B. Signal Modeling for Ion Cyclotron Resonance. J. Chem. Phys. 1978, 69, 4097-4104. 